\title{
The quartic equation: invariants and Euler's solution revealed $\square$
}

RWD Nickalls 2

The Mathematical Gazette (2009); vol.93 (March; No. 526), pp.66-75

http://www.nickalls.org/dick/papers/maths/quartic2009.pdf

\section{Introduction}

The central role of the resolvent cubic in the solution of the quartic was first appreciated by Leonard Euler (1707-1783). Euler's quartic solution first appeared as a brief section $(\S 5)$ in a paper on roots of equations $[1,2]$, and was later expanded into a chapter entitled Of a new method of resolving equations of the fourth degree ( $\$ \S 773-783)$ in his Elements of algebra [3, 4].

Euler's quartic solution was an important advance, in which he showed that each of the roots of a reduced quartic can be represented as the sum of three square roots, say $\pm \sqrt{r_{1}} \pm \sqrt{r_{2}} \pm \sqrt{r_{3}}$, where the $r_{i}(i=1,2,3)$ are the roots of a resolvent cubic. A quartic equation in $x$ is said to be reduced if the coefficient of $x^{3}$ is zero. This can always be achieved by a simple change of variable.

Motivated by the recent tercentenary of Euler's birth, this article describes the geometric basis underlying both the $r_{i}$ and the sign of the product $\sqrt{r_{1} r_{2} r_{3}}$, these being two key aspects of Euler's solution. Finally, we reveal the beautiful dynamic between Euler's resolvent cubic and the quartic invariants $G, H, I, J$ $[5,6,7]$, and propose a new class of algebraic object.

\section{Geometric basis for the $r_{i}$}

A significant property of the reduced quartic equation is that the four roots can be completely defined using only three parameters. For example, let $z_{j}$ $(j=1,2,3,4)$ be the roots (see Figure 1) of a reduced quartic equation,

$$
Z(x) \equiv a x^{4}+p x^{2}+q x+r=0 .
$$

As the sum of the roots is zero (the coefficient of the cubic term is zero), it follows that we can define the points midway between $z_{1}, z_{2}$ and $z_{3}, z_{4}$ as $\pm g$. Let $z_{2}-z_{1}=2 \alpha$ and $z_{4}-z_{3}=2 \beta$. The four roots can then be expressed as follows:

$$
\left\{\begin{array}{l}
z_{1}, z_{2}=-g \pm \alpha \\
z_{3}, z_{4}=+g \pm \beta
\end{array}\right.
$$

Since specifying one pair of quartic roots necessarily defines the remaining pair, there are just three different ways of allocating the pairs of roots, each associated with its own $g, \alpha, \beta$, the inter-relationship between which lies at the heart of a remarkable symmetry which underpins the solution of the quartic.

\footnotetext{
${ }^{1}$ This minor revision of the original article corrects typographic errors and incorporates some explanatory footnotes. The original published version is available from the JSTOR archive at http://www.jstor.org/stable/40378672

${ }^{2}$ Department of Anaesthesia, Nottingham University Hospitals, City Hospital Campus, Nottingham, UK. email: dick@nickalls.org
} 


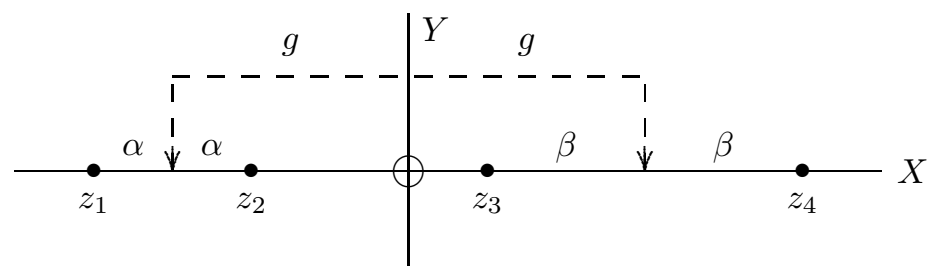

Figure 1:

For example if, with no loss of generality, we let

$$
\left\{\begin{aligned}
z_{3}+z_{4} & =2 g_{1}, \\
z_{3}+z_{1} & =2 g_{2}, \\
z_{3}+z_{2} & =2 g_{3},
\end{aligned}\right.
$$

then

$$
\begin{aligned}
2\left(g_{2}+g_{3}\right) & =2 z_{3}+z_{1}+z_{2}, \\
& =\left(z_{1}+z_{2}+z_{3}+z_{4}\right)+z_{3}-z_{4}, \\
& =z_{3}-z_{4}=-2 \beta_{1},
\end{aligned}
$$

and similarly

$$
2\left(g_{2}-g_{3}\right)=z_{1}-z_{2}=-2 \alpha_{1}
$$

and hence

$$
\left\{\begin{array}{c}
\alpha_{1}=-\left(g_{2}-g_{3}\right), \\
\beta_{1}=-\left(g_{2}+g_{3}\right) .
\end{array}\right.
$$

Thus the $\alpha_{k}, \beta_{k}(k=1,2,3)$ are actually simple functions of the $g_{i}(i \neq k)$ such that each of the four roots $z_{j}$ can be expressed as a function of the $g_{i}$ alone, as follows 3

$$
\left\{\begin{array}{l}
z_{1}=-g_{1}-\alpha_{1}=-g_{1}+\left(g_{2}-g_{3}\right)=-g_{1}+g_{2}-g_{3} \\
z_{2}=-g_{1}+\alpha_{1}=-g_{1}-\left(g_{2}-g_{3}\right)=-g_{1}-g_{2}+g_{3} \\
z_{3}=+g_{1}-\beta_{1}=+g_{1}+\left(g_{2}+g_{3}\right)=+g_{1}+g_{2}+g_{3} \\
z_{4}=+g_{1}+\beta_{1}=+g_{1}-\left(g_{2}+g_{3}\right)=+g_{1}-g_{2}-g_{3}
\end{array}\right.
$$

Thus Euler's $r_{i}$ are the same as the $g_{i}^{2}$.

\section{Euler's resolvent cubic}

Using these observations we can reconstruct a given reduced quartic equation, say Equation 1] which then leads to a resolvent cubic and hence to the solution. Let the roots of $Z(x)=0$ be $-g \pm \alpha$ and $g \pm \beta$ (Figure 1).

$$
Z(x) \equiv\{x-(-g-\alpha)\}\{x-(-g+\alpha)\}\{x-(g-\beta)\}\{x-(g+\beta)\}=0 .
$$

Expanding and letting $A=g^{2}-\alpha^{2}$ and $B=g^{2}-\beta^{2}$, gives

$$
x^{4}+\left(-4 g^{2}+A+B\right) x^{2}+(2 g)(B-A) x+A B=0 .
$$

\footnotetext{
${ }^{3}$ For a 3D version of Euler's solution in which the $\pm g_{i}$ are associated with the mid-points of the six edges of a regular tetrahedron, see Fig 2 in Nickalls (2012), The quartic equation: alignment with an equivalent tetrahedron, Mathematical Gazette, $\underline{96}, 49-55$; http://www.nickalls.org/dick/papers/maths/tetrahedron2012.pdf
} 
We can eliminate $\alpha, \beta$ by first equating coefficients with the monic form of Equation 1 giving

$$
\left\{\begin{aligned}
p / a & =-4 g^{2}+A+B, \\
q / a & =2 g(B-A), \\
r / a & =A B,
\end{aligned}\right.
$$

and then eliminating $A$ and $B$ (using the identity $4 A B=2 A \times 2 B$ ), which generates a resolvent sextic in $g$, the roots of which are the six values $\pm g_{1}, \pm g_{2}, \pm g_{3}$. The substitution $g^{2} \mapsto x$ then generates Euler's original resolvent cubic [1, 2, $3,4]$

$$
R(x) \equiv x^{3}+\frac{p}{2 a} x^{2}+\left(\frac{p^{2}-4 a r}{16 a^{2}}\right) x-\frac{q^{2}}{64 a^{2}}=0,
$$

whose roots $r_{i}$ are therefore $g_{1}^{2}, g_{2}^{2}, g_{3}^{2}$. The four roots of the reduced quartic $Z(x)=0$ are among the eight possible values of $\pm \sqrt{r_{1}} \pm \sqrt{r_{2}} \pm \sqrt{r_{3}}$; but in order to determine which four they are we need a way of allocating the signs correctly.

Euler, using a monic quartic of the form $x^{4}-l x^{2}-m x-n=0$, says he resolved the sign problem by noting that $\sqrt{r_{1} r_{2} r_{3}}=m / 8$, as follows $[3, \S 773]$ :

$\ldots$ But it is to be observed, that the product ... $\sqrt{r_{1} r_{2} r_{3}}$, must be equal to $m / 8$, and that if $m / 8$ be positive, the product of the terms $\sqrt{r_{1}}, \sqrt{r_{2}}, \sqrt{r_{3}}$, must likewise be positive;

Unfortunately Euler did not elaborate further on this, but the key to understanding the sign problem is not difficult to find, since from Equation 2 we have

$$
\begin{aligned}
8 g_{1} g_{2} g_{3} & =\left(z_{3}+z_{4}\right)\left(z_{3}+z_{1}\right)\left(z_{3}+z_{2}\right) \\
& =z_{3}^{3}+z_{3}^{2}\left(z_{1}+z_{2}+z_{4}\right)+z_{3}\left(z_{2} z_{1}+z_{2} z_{4}+z_{1} z_{4}\right)+z_{4} z_{1} z_{2} .
\end{aligned}
$$

Now $z_{1}+z_{2}+z_{4}=-z_{3}\left(\right.$ since $\left.\Sigma z_{j}=0\right)$, hence

$$
8 g_{1} g_{2} g_{3}=z_{1} z_{2} z_{3}+z_{2} z_{3} z_{4}+z_{3} z_{4} z_{1}+z_{4} z_{1} z_{2},
$$

and so $8 g_{1} g_{2} g_{3}$ is actually one of the four elementary symmetric functions of the roots $z_{j}$. Its value is therefore equal to $-1 \times$ the coefficient of the $x$-term of the monic form of the reduced quartic equation $Z(x)=0$, and so we have

$$
8 \sqrt{r_{1} r_{2} r_{3}}=8 g_{1} g_{2} g_{3}=-q / a,
$$

which is equivalent to Euler's $\sqrt{r_{1} r_{2} r_{3}}=m / 8$.

\section{Geometric basis for the sign of $\sqrt{r_{1} r_{2} r_{3}}$}

A useful way of 'seeing' the quartic algebra at work is to express the coefficients in terms of the key 'visible' parameters $\varepsilon, y_{N z}, y_{N z^{\prime}}$ shown in Figure 2, as follows: Let $F(X)$ be a quartic polynomial with real coefficients $(a \neq 0)$

$$
F(X) \equiv a X^{4}+b X^{3}+c X^{2}+d X+e,
$$

with invariants $[6$, p. 76$]$

$$
\left\{\begin{aligned}
G & =b^{3}+8 a^{2} d-4 a b c \\
H & =8 a c-3 b^{2} \\
I & =12 a e-3 b d+c^{2} \\
J & =72 a c e+9 b c d-27 a d^{2}-27 e b^{2}-2 c^{3}
\end{aligned}\right.
$$




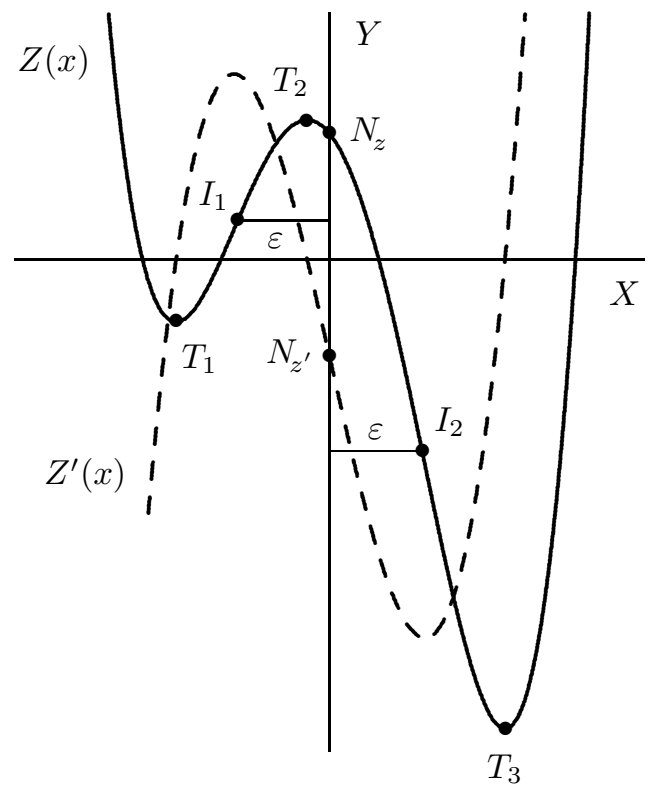

Figure 2:

The reduced quartic $Z(x)$, turning points $\left(T_{1}, T_{2}, T_{3}\right)$, points of inflection $\left(I_{1}, I_{2}\right)$, and first differential $Z^{\prime}(x)$. The $x$-coordinates of the points of inflection are $\pm \varepsilon$. The curves intersect the $y$-axis at points $N_{z}$ and $N_{z^{\prime}}$.

Let its reduced form $Z(x)$ be generated by the translation $X \mapsto x+X_{N f}$, where $X_{N f}=-b /(4 a)$. Using Taylor's theorem we have

$$
Z(x) \equiv F\left(x+X_{N_{f}}\right)=a x^{4}+\frac{F^{\prime \prime}\left(X_{N_{f}}\right)}{2} x^{2}+F^{\prime}\left(X_{N_{f}}\right) x+F\left(X_{N_{f}}\right) .
$$

If $Z(x)$ and $Z^{\prime}(x)$ intersect the $y$-axis in points $N_{z}$ and $N_{z^{\prime}}$ respectively, then Equation 8 can be expressed as

$$
Z(x) \equiv a x^{4}-6 a \varepsilon^{2} x^{2}+y_{N z^{\prime}} x+y_{N z}
$$

where (see Equation 4 and Figures 2,3

$$
\left\{\begin{aligned}
\varepsilon^{2} & =\frac{\left(3 b^{2}-8 a c\right)}{48 a^{2}} \equiv \frac{-H}{48 a^{2}} \equiv \frac{-p}{6 a}, \\
y_{N z} & =F\left(X_{N f}\right) \equiv \frac{I}{12 a}-\frac{3 H^{2}}{48^{2} a^{3}} \equiv r, \\
y_{N z^{\prime}} & =F^{\prime}\left(X_{N f}\right) \equiv \frac{G}{8 a^{2}} \equiv q, \\
-12 a \varepsilon^{2} & =F^{\prime \prime}\left(X_{N f}\right) .
\end{aligned}\right.
$$

Expressing the reduced quartic $Z(x)$ in this form (Equation 9 greatly facilitates visualisation, since we can now 'see' how the configuration of the curves $Z(x)$ and $Z^{\prime}(x)$ is related to the coefficients. For example (assuming $a>0$ ), if the $x^{2}$ term is positive then $\varepsilon$ is complex $\left(\varepsilon^{2}<0\right)$, and so the quartic will have two complex points of inflection and hence only one real turning point (cf. [10]). 
If $x_{T i}$ are the $x$-coordinates of the turning points of $Z(x)$, then by differentiating Equation 9 we have (see Equations $5 a$ and 10 )

$$
4 x_{T_{1}} x_{T_{2}} x_{T_{3}}=\frac{-y_{N z^{\prime}}}{a}=8 \sqrt{r_{1} r_{2} r_{3}}
$$

and hence the sign of $\sqrt{r_{1} r_{2} r_{3}}$ is the same as that of $-y_{N z^{\prime}} / a$ and $x_{T_{1}} x_{T_{2}} x_{T_{3}}$. It follows, therefore, that we can actually 'see' the correct sign of $\sqrt{r_{1} r_{2} r_{3}}$ simply by observing the signs of the abscissae of the turning points of the reduced quartic, or by noting the location of $N_{z^{\prime}}$ in relation to the abscissa.

For example (assuming $a>0$ ), if the roots $z_{j}$ are such that the middle turning point, $T_{2}$, is to the left of the $y$-axis, then not only will $y_{N z^{\prime}}$ be negative (Figure 2 but just two of the three $x_{T_{i}}$ will be negative resulting in a positive product for $x_{T_{1}} x_{T_{2}} x_{T_{3}}$, and hence $\sqrt{r_{1} r_{2} r_{3}}$ will also be positive (see Equation 11). Conversely, if the middle turning point is to the right of the $y$-axis, then $y_{N z^{\prime}}$ will be positive, and only one of the $x_{T_{i}}$ will be negative making the product $x_{T_{1}} x_{T_{2}} x_{T_{3}}$ negative.

\section{Roots}

As regards the roots $z_{j}$ of the reduced quartic $Z(x)$, we can initially choose any sign combination for the $\sqrt{r_{i}}$, and then evaluate the sign of the product $\sqrt{r_{1} r_{2} r_{3}}$. If the sign of the product is the same as that of $-y_{N z^{\prime}} / a$ (see Equation 11) then we have a valid combination of signs, and can proceed to determine the four $z_{j}$ using Equation 3. Otherwise, it is only necessary to change the sign of any one of the $\sqrt{r_{i}}$ (say, $\sqrt{r_{1}} \rightarrow-\sqrt{r_{1}}$ ), and proceed as before using Equation 3

When the reduced quartic is symmetric about the $y$-axis one of the $x_{T_{i}}$ will be zero and hence the product $\sqrt{r_{1} r_{2} r_{3}}$ is zero. However, the solution in this case is trivial since $Z(x)$ is then an even function as $y_{N z^{\prime}}$ is also zero.

\section{Application}

Since all resolvent cubics of the quartic can be transformed to a standard form [9], typically expressed as $[6$, p. 77$]$

$$
T(x) \equiv x^{3}-3 I x+J,
$$

we can solve any quartic by solving instead a simple reduced form of the resolvent, say $T(x)=0$, and then recover the roots of Euler's resolvent using the transformation which carries the reduced form back to $R(x)$.

For example, the translation $x \mapsto x+x_{N r}$ to reduce $R(x)$, for which $x_{N r}=-p /(6 a) \equiv \varepsilon^{2}$, generates the reduced form $S(x)$, as follows:

$$
S(x) \equiv R\left(x+\varepsilon^{2}\right) \equiv x^{3}-\frac{I}{48 a^{2}} x+\frac{J}{1728 a^{3}} .
$$

The substitution $x \mapsto x /(12 a)$ then scales $1728 a^{3} S(x)$ to $T(x)$, and hence if the roots of $S(x)=0$ and $T(x)=0$ are $s_{i}$ and $t_{i}$ respectively, then

$$
r_{i}=s_{i}+\varepsilon^{2}=\frac{t_{i}}{12 a}+\varepsilon^{2} .
$$

This convenient approach is illustrated in Example 1. 


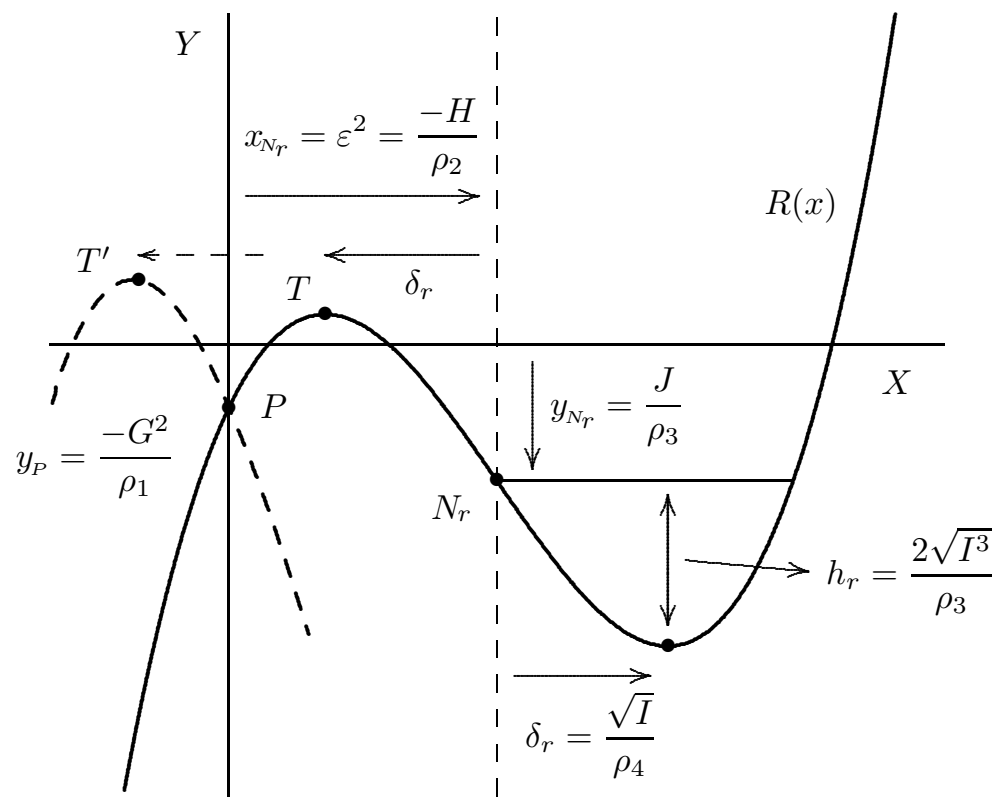

Figure 3:

Euler's resolvent cubic $R(x)$ with three real roots $\left(h_{r}^{2}>y_{N_{r}}^{2}\right.$, i.e. $\left.4 I^{3}>J^{2}\right)$ which are all positive $\left(\varepsilon^{2}>0, x_{N_{r}}^{2}>\delta_{r}^{2}\right)$. The conditions $\varepsilon^{2}>0, x_{N_{r}}^{2}<\delta_{r}^{2}$ are associated with two negative roots (dashed curve). Note that $G^{2}$, $H, I, J$ are constant multiples respectively of the resolvent's geometric parameters $y_{P}, x_{N_{r}}, \delta_{r}^{2}, y_{N_{r}}\left(\rho_{1}=64^{2} a^{6}, \rho_{2}=48 a^{2}, \rho_{3}=1728 a^{3}, \rho_{4}=12 a\right)$.

The invariants $I, J$ are readily visualised since any reduced cubic can be expressed in terms of its geometric parameters $\delta$ and $y_{N}$ as in [8]

$$
A x^{3}-3 A \delta^{2} x+y_{N}=0 .
$$

For example, equating coefficients between $S(x), T(x)$ and the monic form of Equation 15 and noting that $h^{2}=4 A^{2} \delta^{6}[8]$, shows that $I, J$ are simply constant multiples of $\delta^{2}, y_{N}$ as follows (Figure 3 :

$$
\left\{\begin{aligned}
A_{r} & =A_{s}=A_{t}=1, \\
I & =\delta_{r}^{2}(12 a)^{2}=\delta_{s}^{2}(12 a)^{2}=\delta_{t}^{2}, \\
J & =y_{N_{r}}(12 a)^{3}=y_{N_{s}}(12 a)^{3}=y_{N_{t}}, \\
\frac{4 I^{3}}{J^{2}} & =\left(\frac{h_{r}}{y_{N r}}\right)^{2}=\left(\frac{h_{s}}{y_{N s}}\right)^{2}=\left(\frac{h_{t}}{y_{N t}}\right)^{2} .
\end{aligned}\right.
$$

Thus each of these invariants has a visible geometric interpretation in relation to Euler's resolvent cubic, either as a position parameter with respect to the axes $(G, H, J)$, or as a shape parameter $(I)$. For example, we can now see that the condition $J=0$ simply indicates that the $N$-point of the resolvent cubic lies on the $x$-axis and all that that implies (see Example 2). Similarly, the condition $I=0$ indicates that the resolvent adopts the 'cubic parabola' form. Furthermore $y_{P} \leq 0$, which reveals how and why the resolvent cubic cannot have just a single negative root ${ }^{4}$ The syzygy $-27 G^{2}=H^{3}-48 a^{2} I H+64 a^{3} J[6$, p. 76] is generated by substituting into $S(x)$ the coordinates of $P\left(H /\left(48 a^{2}\right),-G^{2} /\left(64^{2} a^{6}\right)\right)$.

\footnotetext{
${ }^{4}$ For real coefficients $G^{2} \geq 0$, and hence $P$ must always be on or below the $x$-axis.
} 


\section{Euler's cubic and the quartic root configurations}

A very significant but seemingly overlooked aspect of Euler's resolvent cubic is its beautiful and symmetric relationship with two important algebraic objects, namely the discriminant $4 I^{3}-J^{2}$ and the seminvariant $H^{2}-16 a^{2} I$, the signs of which distinguish between the various quartic root configurations $[5, \S 68$; 6, p. 80; 7, p. 28]. Visualising the resolvent in relation to the invariants (Figure 3) reveals the mechanisms, as follows:

\section{$7.14 I^{3}-J^{2}$}

Since $h^{2}=4 A^{2} \delta^{6}[8]$, it follows from Equation 16 that

$$
\frac{-\left(4 I^{3}-J^{2}\right)}{12^{6} a^{6}}=y_{N r}^{2}-h_{r}^{2} .
$$

Thus the quartic discriminant $4 I^{3}-J^{2}$ is simply a constant multiple of $y_{N_{r}}^{2}-h_{r}^{2}$, the sign of which reflects whether the $x$-axis lies between $\left(y_{N_{r}}^{2}<h_{r}^{2}\right)$, on $\left(y_{N r}^{2}=h_{r}^{2}\right)$, or outside $\left(y_{N_{r}}^{2}>h_{r}^{2}\right)$ the turning points of the resolvent cubic (Figure 3 .

\section{$7.2 \quad H^{2}-16 a^{2} I$}

The sign of this algebraic object distinguishes (when $\varepsilon^{2}>0$ ) between the then two possible quartic root configurations associated with the case $4 I^{3}-J^{2}>0$, namely (a) four real roots $\left(H^{2}-16 a^{2} I>0\right)$, and (b) four complex roots $\left(H^{2}-16 a^{2} I<0\right)$ [5, §68]. Substituting for $H$ (Equation 10) and $I$ (Equation 16) gives

$$
H^{2}-16 a^{2} I=\left(-48 a^{2} \varepsilon^{2}\right)^{2}-16 a^{2}\left(12^{2} a^{2} \delta_{r}^{2}\right)=3^{2} 4^{4} a^{4}\left(\varepsilon^{4}-\delta_{r}^{2}\right) .
$$

But $\varepsilon^{2}=x_{N r}$ (Figure 3) and hence

$$
\frac{H^{2}-16 a^{2} I}{3^{2} 4^{4} a^{4}}=x_{N r}^{2}-\delta_{r}^{2} .
$$

Thus $H^{2}-16 a^{2} I$ is just a constant multiple of $x_{N_{r}}^{2}-\delta_{r}^{2}$, the sign of which (when $\varepsilon^{2}>0$ ) reflects whether the $y$-axis lies between $\left(x_{N_{r}}^{2}<\delta_{r}^{2}\right)$, on $\left(x_{N_{r}}^{2}=\delta_{r}^{2}\right)$, or outside $\left(x_{N r}^{2}>\delta_{r}^{2}\right)$ the turning points of the resolvent cubic (cf. [6, p. 80, proposition 7]).

For example (Figure 3), when a quartic with three real turning points $\left(\varepsilon^{2}>0\right)$ has four real roots $\left(4 I^{3}-J^{2}>0\right)$ Euler's cubic $R(x)$ has three positive real roots - the $y$-axis lies outside the two turning points - and so $x_{N_{r}}^{2}>\delta_{r}^{2}$ and hence $H^{2}-16 a^{2} I>0$.

Conversely, when a quartic with three real turning points $\left(\varepsilon^{2}>0\right)$ has four complex roots $\left(4 I^{3}-J^{2}>0\right), R(x)$ then has exactly two negative real roots, and so its turning point $T^{\prime}$ (Figure 3 lies to the left of the $y$-axis $\left(x_{N r}^{2}<\delta_{r}^{2}\right.$ ), hence $H^{2}-16 a^{2} I<0$.

\subsection{A new class of object?}

Since $H^{2}-16 a^{2} I$ functions with regard to the $y$-axis in exactly the same way that $4 I^{3}-J^{2}$ functions with regard to the $x$-axis, I would like to suggest that this pair of algebraic objects should be regarded as forming a distinct class of object - thereby linking two previously independent algebraic quantities with a single unifying concept. 


\section{Example 1}

Solve $f(X) \equiv X^{4}-11 X^{3}+41 X^{2}-61 X+30=0$.

The key parameters are: $a=1, X_{N f}=11 / 4, Y_{N f^{\prime}}=f^{\prime}\left(X_{N f}\right)=-15 / 8, G=-15$, $I=28, J=-160, \varepsilon^{2}=35 / 48$. Using say, $T(x)$, we solve ${ }^{5}$

$$
T(x) \equiv x^{3}-84 x-160=0,
$$

the three $t_{i}$ being $-8,-2,10$. The $\sqrt{r_{i}}$ are therefore given by

$$
\left\{\begin{array}{l}
\sqrt{r_{1}}=\sqrt{\varepsilon^{2}+\frac{t_{1}}{12 a}}=\sqrt{\frac{35}{48}-\frac{8}{12}}=\frac{1}{4} \\
\sqrt{r_{2}}=\sqrt{\varepsilon^{2}+\frac{t_{2}}{12 a}}=\sqrt{\frac{35}{48}-\frac{2}{12}}=\frac{3}{4} \\
\sqrt{r_{3}}=\sqrt{\varepsilon^{2}+\frac{t_{3}}{12 a}}=\sqrt{\frac{35}{48}+\frac{10}{12}}=\frac{5}{4}
\end{array}\right.
$$

Since the sign of $-Y_{N f^{\prime}} / a$ is positive ${ }^{6}$ then the product of the $\sqrt{r_{i}}$ must also be positive - which it is. Finally, adding $X_{N_{f}}$ recovers the quartic roots $\left(X_{j}=X_{N f} \pm \sqrt{r_{1}} \pm \sqrt{r_{2}} \pm \sqrt{r_{3}}\right)$ using (3) as follows:

$$
\left\{\begin{array}{l}
X_{1}=\frac{11}{4}-\frac{1}{4}+\frac{3}{4}-\frac{5}{4}=2 \\
X_{2}=\frac{11}{4}-\frac{1}{4}-\frac{3}{4}+\frac{5}{4}=3 \\
X_{3}=\frac{11}{4}+\frac{1}{4}+\frac{3}{4}+\frac{5}{4}=5 \\
X_{4}=\frac{11}{4}+\frac{1}{4}-\frac{3}{4}-\frac{5}{4}=1
\end{array}\right.
$$

Even the solution of $T(x)=0$ is greatly simplified since $\delta, h, y_{N}$ are simple functions of $I$ and $J$ (see Equation 16 ). For example, $T(x)=0$ has three real roots in this case since $\left(y_{N t} / h_{t}\right)^{2} \equiv J^{2} /\left(4 I^{3}\right) \leq 1[8]$.

\section{$9 \quad$ Example 2}

Explain the significance of $J=0, I>0$, for a quartic with four real roots.

The condition $J=0$ implies that Euler's resolvent cubic has its $N$-point on the $x$-axis (Figure 3), and hence it has three roots in arithmetic progression. If also $I>0$ (resolvent cubic has two real turning points), then the resolvent's roots are distinct and (with the root at infinity) form a harmonic range. Since the roots of the parent quartic have the same cross-ratio they also form a harmonic range.

${ }^{5}$ Note that we could instead solve $S(x)=0$, and then use $r_{i}=\varepsilon^{2}+s_{i}$ (see Equation 14).

${ }^{6}$ Since $Y_{N_{f^{\prime}}} \equiv G /\left(8 a^{2}\right)$ it is probably more convenient to use the sign of $-G / a$ instead. 


\section{Acknowledgements}

I would like to thank Professor JE Cremona (University of Warwick) for reading drafts of this paper and for his constructive comments. I also acknowledge the helpful suggestions of the anonymous reviewer.

\section{References}

1 L. Euler. De formis radicum aequationum cujusque ordinis conjectatio. Commentarii academiae scientiarum imperialis Petropolitianae (1733); 6 (pub. 1738), pp. 216-231 = Opera Omnia, Series 1 (Pure mathematics), vol. 6 (Theory of equations), pp.1-19. [Euler Archive, E30 (Latin): http://www.eulerarchive.org/pages/E030.html

2 Bell J (2008). A conjecture on the forms of the roots of equations. arXiv:0806.1927v1 [math.HO]. http://arxiv.org/abs/0806.1927][An English translation of Euler's De formis radicum aequationum cujusque ordinis conjectatio (E30)].

3 L. Euler. Vollständige Anleitung zur Algebra (Elements of algebra), 2 vols, Royal Academy of Sciences, St. Petersburg (1770).

[Euler Archive, E387 (English): http://www.eulerarchive.org/docs/originals/E387e.P1S4.pdf

4 C. R. Sangwin (Ed.). Euler's Elements of Algebra. Tarquin Publications, St Albans, UK (2006). [English translation of Euler 1770 (E387)]

5 W. S. Burnside and A. W. Panton. The theory of equations: with an introduction to the theory of binary algebraic forms. (7th edn.) 2 vols; Longmans, Green and Co., London (1912).

6 J. E. Cremona. Reduction of binary cubic and quartic forms. J. Comput. Math., 2 (1999), pp.62-92. http://www.lms.ac.uk/jcm/2/lms98007/ [The seminvariants $G$ and $H^{2}-16 a^{2} I$ are denoted here by $R$ and $3 Q$ respectively]

7 P. J. Olver. Classical invariant theory. London Mathematical Society Student Texts No. 44. Cambridge University Press (1999).

8 R. W. D. Nickalls. A new approach to solving the cubic: Cardan's solution revealed. Math. Gaz., 77 (Nov 1993) pp. 354-359.

http://www.nickalls.org/dick/papers/maths/cubic1993.pdf. http://www.jstor.org/stable/3619777

9 R. S. Ball. Note on the algebraical solution of biquadratic equations. Quarterly Journal of Pure and Applied Mathematics, 7 (1866) pp. 6-9, 358369. http://www.nickalls.org/dick/papers/maths/ball1866quartic.pdf

10 J. P. Dalton. On the graphical discrimination of the cubic and of the quartic. Math. Gaz., 17 (July 1933) pp. 189-196

http://www.jstor.org/stable/3607613 